\title{
ACTIVE REHABILITATION FOR CHRONIC LOW BACK PAIN
}

\section{$4 \underline{\text { Abstract }}$}

5 Patients with low back pain often demonstrate elevated paraspinal muscle activity

6 compared to asymptomatic controls. This hyperactivity has been associated with a

7 delayed rate of stature recovery following spinal loading tasks. The aim of this study

8 was to investigate the changes in muscle activity and stature recovery in patients with

9 chronic low back pain following an active rehabilitation programme. The body height

10 recovery over a 40-minute unloading period was assessed via stadiometry and surface

11 electromyograms were recorded from the paraspinal muscles during standing. The

12 measurements were repeated after patients had attended a rehabilitation programme and

13 again at a six-month follow-up. Analysis was based on 17 patients who completed the

14 post-treatment analysis and 12 of these who also participated in the follow-up. By the

15 end of the six months, patients recovered significantly more height during the unloading

16 session than at their initial visit $(\mathrm{ES}=1.18 ; \mathrm{P}<0.01)$. Greater stature recovery

17 immediately following the programme was associated with decreased pain $(r=-0.55 ; \mathrm{P}$

$18=0.01)$. The increased height gain after six months suggests that delayed rates of

19 recovery are not primarily caused by disc degeneration. Muscle activity did not decrease

20 after treatment, perhaps reflecting a period of adaptation or altered patterns of motor

21 control.

$23 \underline{\text { Key Words }}$

24 Low back pain; electromyography; stature change 


\section{$1 \quad$ Introduction}

2 Intervertebral discs lose height in response to compressive forces, due to a combination of

3 fluid outflow and elastic deformation of both the disc and the vertebral endplates. When the

4 spine is subsequently unloaded, these processes are reversed, leading to elastic return, fluid

5 inflow and disc height recovery (Adams et al., 1990). Changes in disc height lead to

6 changes in overall body length (or stature). Therefore precision stadiometry, which

7 measures changes in body height, is often used as an indirect and non-invasive method for

8 assessing changes in disc height and comparing the relative spinal loading resulting from

9 different activities. It has been observed that people with chronic low back pain (CLBP)

10 appear to lose stature at a similar rate to healthy controls in response to loading, but are

11 significantly slower to recover this height when the spine is unloaded (Rodacki et al., 2003;

12 Healey et al., 2005). Possible causes of this may be an altered response due to disc

13 degeneration (Urban \& Roberts, 2003), or elevated muscle activity increasing the

14 compressive forces acting on the spine (Healey et al., 2005).

16 Increased activity of the superficial paraspinal muscles during static postures such as

17 standing (Ambroz et al., 2000) or full flexion (Watson et al., 1997b) is often reported in

18 patients with CLBP. It is unknown why this hyperactivity occurs, although it may

19 reflect a compensatory mechanism in the presence of spinal instability (possibly caused

20 by injury, disease or degeneration) (Panjabi, 1992), and may persist after the original

21 injury or cause has disappeared (van Dieën et al., 2003).

23 Healey et al. (2005) found a significant negative correlation between paraspinal muscle

24 activity and stature recovery in people with mild CLBP, suggesting that the increased

25 muscle activity may increase the loading on the intervertebral discs and delay their 
1 regain of height. This is of clinical consequence because intervertebral disc height loss may

2 compromise spinal stability (Zhao et al., 2005), increase loading on other spinal structures,

3 such as the facet joints, and lead to concentrations of compressive stress (Adams et al.,

4 2002). Significant correlations have been observed between delayed stature recovery and

5 higher levels of both pain and disability (Healey et al., 2005), supporting the relevance of

6 this research area.

7

8 Treatment programmes can affect both the activity of the superficial back muscles and

9 stature recovery in patients with low back pain (LBP). For example, reduced muscle

10 activity at full flexion has been observed following a pain management programme

11 (Watson et al., 1997a) and an intense physical exercise rehabilitation programme has

12 been shown to significantly increase the morning height of patients compared to those

13 who received no treatment (Hupli et al., 1997). Reduced muscle contraction was

14 suggested as a possible explanation in this case.

16 The aim of this study was to investigate the changes in muscle activity and stature

17 recovery following an active rehabilitation programme and to establish if there was any

18 relationship with clinical outcome. It was hypothesized that muscle activity would be

19 reduced following the programme and that this would be associated with increases in

20 stature recovery and also with improvements in pain and disability.

$22 \underline{\text { Methods }}$

23 Participants

24 Patients with CLBP (LBP lasting more than three months) were recruited from the 25 waiting lists for both the Back Exercise Group (BEG) and the Work Back to Life Group 
1 (WBTL), both of which are run in [removed to maintain anonymity]. Patients on these

2 waiting lists were sent information about the study in the post and asked to return a

3 reply slip if they were willing to take part. All testing of NHS patients took place at

$4 \quad$ [removed to maintain anonymity].

5

6 Exclusion criteria were; nerve root compression, central nervous system impairment,

7 progressive motor deficit, sphincter impairment from neurologic cause and presence of

8 "red flags" (e.g. unexplained weight loss, recent urinary tract infection, history of

9 intravenous drug use). Many of the patients were taking analgesics for their back pain; it

10 was not considered practical, or ecologically valid, to exclude those on medication.

11 Participants were offered $£ 12.50$ for each session they attended to cover travel and

12 parking expenses. Ethical approval was granted by the [removed to maintain anonymity]

13 NHS ethics committee and local NHS permission was granted by [removed to maintain

14 anonymity] NHS Trust. All participants provided written informed consent.

16 Muscle Activity Measurement

17 Raw electromyographic (EMG) signals were recorded using a DELSYS system (Delsys

18 Inc. Boston, MA, USA). Single differential surface electrodes consisting of two silver

19 bars with an inter-electrode spacing of $10 \mathrm{~mm}$ were used. Signals were band-pass

20 filtered between 20 and $450 \mathrm{~Hz}$ with a sampling frequency of $1000 \mathrm{~Hz}$. Electrodes were

21 placed over the erector spinae muscle at the level of the L1-2 and L4-5 interspaces,

22 approximately $3 \mathrm{~cm}$ from the midline on either side and a reference electrode placed on

23 the right iliac crest. Participants assumed a standing posture for ten seconds while a

24 recording was taken. EMG data were normalised relative to a reference voluntary 
1 contraction (RVC). A sub-maximal RVC was used as this has been shown to increase

2 between-day reliability within CLBP patients (Dankaerts et al., 2000). The reference

3 task required each participant to stand while holding up a $0.5 \mathrm{~kg}$ mass in each hand with

4 arms bent (upper arms horizontal, lower arms vertical) for ten seconds. The signal mean

5 value was removed from the raw EMGs, before rectifying and integrating over a period

6 of five seconds. An analysis of variance showed no significant difference between the

7 EMG data at the different electrode sites and hence an average of the four sites was used

8 in the analysis. The EMG reading and the RVC were both taken to be the average of the

9 three readings recorded during the session. The non-normalised values were also

10 analysed but, unless specified, muscle activity refers to values normalised to the RVC.

12 The stadiometer

13 Changes in stature were measured with a standing stadiometer, which consisted of a

14 rigid frame, mounted at a right angle to a base plate and inclined backward $15^{\circ}$ from the

15 vertical (Figure 1). Four anatomical points were identified (Lewis \& Fowler, 2009) and

16 then supported by the frame to maintain the natural contours of the head and spine. The

17 position of the feet was marked and head position was controlled by the use of spectacle

18 frames with attached lasers, aligned with two movable targets above the participant's

19 head. A high-resolution linear variable displacement transducer (Solartron Metrology,

20 DC50) was used to detect changes in stature by measuring vertical displacement with an

21 accuracy of approximately $0.01 \mathrm{~mm}$. The information was observed graphically on a

22 laptop computer at the time of collection and stored digitally, at a sampling rate of

$23100 \mathrm{~Hz}$, for later analysis. 
1 All participants initially undertook a brief familiarisation session on the stadiometer to

2 enable them to practice the adoption of a repeatable and comfortable posture. This

3 consisted of five recordings, between which the participant was asked to lean forward

4 and break contact with the postural controls before resuming their position for the next

5 measurement. A pilot study demonstrated that this approach was sufficient to produce

6 reliable stadiometer readings (average standard deviation (SD) $1.0 \mathrm{~mm}$, standard error of

7 measurement $0.8 \mathrm{~mm}$ ). Participants remained in position for a period of 20 seconds and

8 the stature value used was the mean reading over the final 10 seconds.

9

10 Pain intensity

11 A numerical rating scale (NRS) was employed to assess pain intensity. Participants

12 were asked to rate their pain during the past 24 hours on a scale ranging from (0) 'no

13 pain' to (10) 'worst possible pain. Research supports the reliability and validity of

14 numerical rating scales of pain intensity (Jensen, 2003).

16 Disability

17 The Roland Disability Questionnaire (Roland and Morris, 1983) (RDQ) is a 24-item

18 self-report measure that assesses disability due to back pain. Patients are asked to select

19 which statements, related to perceived limitations in typical daily activities, apply to

20 them. The RDQ has excellent reliability, validity and responsiveness (Roland and

21 Fairbank, 2000; Turner et al., 2003). 
1 A number of additional questionnaires were included to assess psychological factors.

2 The questionnaires used were the Hospital Anxiety and Depression Scale (HADS)

3 (Zigmond and Snaith, 1983), the functional subscale of the Chronic Pain Self-Efficacy

4 Scale (CPSS-PF) (Anderson et al., 1995), the Tampa Scale of Kinesiophobia (TSK)

5 (Kori et al., 1990), the Pain Catastrophising Scale (PCS) (Sullivan et al., 1995) and the

6 Pain Anxiety Symptoms Scale-20 (PASS-20) (Coons et al., 2004).

7

\section{$8 \quad$ Procedure}

9 Patients attended their first testing session (Session 1) before starting the rehabilitation

10 programme (except for five patients who attended in the week after the programme

11 commenced). Patients then returned for another testing session (Session 2) as soon as

12 practical (usually within the following week and always within the following fortnight)

13 after completing the rehabilitation group and then again six months later (Session 3),

14 where possible. Patients were requested to try to maintain the same daily routine prior to

15 attending each testing session in order to reduce fluctuations in physical activity levels.

16 Although it was not possible for all patients to be tested at the same time of day, each

17 patient attended at the same time for each of their visits (or within one hour of their

18 previous time), apart from one exception when, due to work commitments, the patient

19 attended in the morning for Session 1 and in the afternoon for Sessions 2 and 3.

21 For each session, a baseline stature measurement and initial EMG readings (at rest and

22 during the RVC) were taken before participants assumed an unloading position on a

23 physiotherapy bed for 20 minutes (either a side-lying or prone position). After 20

24 minutes, the participants stood up and performed the same EMG and stadiometer

25 measurements, before again assuming an unloading position for a further 20 minutes. 
1 The measurements were then taken for a final time. Stature change was calculated as the

2 difference between the final and the initial stadiometer readings. At the end of the

3 testing session patients were asked about their pain intensity during the past 24 hours.

4 They were then given a questionnaire booklet containing the self-report measures.

5 Although some patients completed the booklet immediately, the majority completed it

6 at home and returned it at a later date.

7

$8 \quad$ Study population

9 Twenty-three patients attended both Sessions 1 and 2 (Table 1). Some patients found it

10 difficult to maintain a consistent posture in the stadiometer and four patients were

11 excluded from the stature recovery data as the SD of the five familiarisation readings

12 was considered too high. For this purpose, a SD of $1.7 \mathrm{~mm}$ was taken as the cut-off point

13 (Lewis et al., 2012). The remaining 19 patients had an average SD of $1.1 \mathrm{~mm}$ over the

14 five familiarisation readings. The stature recovery data of one patient was excluded as

15 he was considered to be an outlier and one further patient had incomplete EMG data

16 resulting from technical problems. The analysis was therefore based on 17 patients

17 (Table 1). Three patients did not complete the questionnaire booklet on both visits and

18 so the data for disability and psychological factors are based on 14 patients.

20 Thirteen patients participated in all three sessions. After excluding one outlier (as

21 above), the follow-up analysis was based on 12 patients (Table 1). Two patients did not

22 complete the questionnaire booklet on both visits and hence the analysis for disability

23 and psychological factors is based on 10 patients. Lewis (2011) provides further details

24 and analysis regarding the drop-outs from this study. 
3 Two active, physiotherapy based interventions were utilised for the purposes of this

4 study. The Back Exercise Group (BEG) involved four sessions (one a week). The first

5 and last sessions were two hours in duration and consisted of exercise and education.

6 The middle two sessions were one hour of exercise only. The exercise facet of the

7 programme consisted of specific stretching and strengthening exercises and became

8 progressively more difficult over the four weeks. Patients were also encouraged to

9 exercise daily at home. The Work Back to Life (WBTL) group included five sessions

10 (one a week), each of three and a quarter hours in duration. This programme included

11 the exercise and education components that were in the BEG, but was based more on

12 cognitive-behavioural principles. In particular, the WBTL group included individual

13 goal setting aimed at returning patients to activities and tasks that they had stopped

14 doing because of their back pain. Patients were allocated to either the BEG or the

15 WBTL based on the results of TSK and RDQ questionnaires, with the WBTL group

16 intended for those patients who were more severely disabled and demonstrated

17 psychosocial risk factors for prolonged disability. Further details on the WBTL

18 programme are given in the study by Woby et al. (2008). As the current study was not

19 aiming to investigate the efficacy of specific interventions, the analysis was carried out

20 on the BEG $(n=16)$ and WBTL $(n=7)$ groups combined.

22 Analysis

23 Parametric tests were used based on the results of Kolmogorov-Smirnov and Shapiro-

24 Wilk tests of normality. One-tailed paired t-tests were performed to identify any pre- to 
1 post- treatment changes and Pearson's correlation coefficient was implemented to

2 determine the inter-relations that existed between the changes in the outcome measures.

3 Effect sizes (difference in means divided by initial SD) were also calculated to provide

4 an indication of the meaningfulness of any changes that occurred. Finally, two-tailed

5 correlation coefficients were employed to investigate the extent to which any of the

6 measures at baseline were linked to changes in muscle activity and stature recovery.

$8 \quad \underline{\text { Results }}$

$9 \quad$ Immediately following treatment

10 A summary of the main outcome measures before (Session 1) and immediately

11 following (Session 2) treatment are given in Table 2. Overall, there were significant

12 improvements in both pain and disability immediately after the programmes. There was

13 also a trend for greater stature recovery, but this did not reach significance $(\mathrm{P}=0.08)$.

14 Changes in stature recovery between Sessions 1 and 2 were correlated with changes in

15 pain $(\mathrm{r}=-0.55, \mathrm{P}=0.01)$ and catastrophising $(\mathrm{r}=-0.65, \mathrm{P}<0.01)$, with a trend for a

16 correlation with changes in disability $(\mathrm{r}=-0.40, \mathrm{P}=0.08)$. Two-tailed analysis showed

17 a trend for patients with higher EMG levels after the programme to be those with higher

18 baseline self-efficacy $(\mathrm{r}=0.52, \mathrm{P}=0.06)$.

$20 \quad$ Follow-up analysis

21 The results for the patients who completed the six-month follow-up (Session 3) are

22 given in Table 3. Stature recovery was significantly greater at Session 3 than at Session

$231(\mathrm{ES}=1.18, \mathrm{P}<0.01)$ and disability was significantly reduced $(\mathrm{ES}=-0.59, \mathrm{P}<0.05)$.

24 There were significant correlations between changes in muscle activity levels between 
1 Sessions 1 and 3 and changes in each of disability $(r=0.61, P=0.03)$, catastrophising $(r$

$2=0.85, \mathrm{P}<0.01)$, pain-related anxiety $(\mathrm{r}=0.69, \mathrm{P}=0.01)$, depression $(\mathrm{r}=0.59, \mathrm{P}=$

$30.04)$ and self-efficacy $(r=-0.57, P=0.04)$, although it should be remembered that

4 these analyses were based on 10 patients only. No association was found between

5 changes in muscle activity and changes in stature recovery. A reduction in EMG by the

6 end of the six-month follow-up period was correlated with high initial levels of muscle

7 activity $(\mathrm{r}=-0.60, \mathrm{P}=0.04)$.

8

9 Discussion

10 Stature recovery was significantly increased at the follow-up session (to levels

11 comparable with asymptomatic individuals (Lewis, 2011)), suggesting that the reduced

12 stature recovery previously observed in patients with CLBP (Healey et al., 2005) is not

13 primarily the result of disc degeneration. This is consistent with a study carried out by

14 Hupli et al. (1997), in which the morning height of patients increased after an intense

15 physical exercise programme, with no observed changes in markers of disc

16 degeneration. In the current study, on average, patients gained an additional $1.9 \mathrm{~mm}$ in

17 height during the unloading period at the follow-up compared to their initial visit,

18 representing an increase of $73 \%$. The increase in recovery also exceeds the standard

19 error of measurement of $1.4 \mathrm{~mm}$ assessed via an earlier repeatability study (Lewis,

20 2011). This involved ten participants from the same patient population as the current

21 study, with stature recovery measurements taken on two separate days, both before the

22 patient commenced the rehabilitation programme. Research into the occurrence and

23 consequences of delayed stature recovery rates within patient groups is limited

24 (providing the motivation for this study). The clinical significance of this change is 
1 therefore unclear, but it seems reasonable to suggest that such enhanced recovery of

2 intervertebral disc height would reduce the loading on other spinal structures and so

3 may facilitate a reduction in symptoms.

5 Immediately following the programme, changes in stature recovery were negatively

6 correlated with changes in pain, with a trend for a link with changes in disability. This

7 suggests that, over periods of up to six weeks, stature recovery measurements could

8 potentially be used as a proxy indicator of changes in clinical outcome and could therefore

9 provide an objective means of assessing progress in patients with back pain.

11 Overall, there was no change in resting EMG immediately following the programme

12 and some patients surprisingly exhibited an increase in muscle activity levels. This

13 pattern existed in both absolute and normalised EMG levels and therefore was not

14 simply due to a reduction in RVC values. This may indicate an adaptation period

15 immediately following a programme of increased activity and exercise, as the muscles

16 compensate for increased demands, possibly in the context of pre-existing instability.

17 There was a trend for increased EMG levels to be associated with higher initial self-

18 efficacy, which may suggest greater participation in the daily exercise and stretching

19 recommended in the programmes. This is not the first study to find that EMG levels do

20 not make an immediate return to more "healthy" patterns of activity. For example,

21 Mannion et al. (2001) reported that a reduction in pain after treatment was not

22 accompanied by increased relaxation of the back muscles during full flexion.

23 Furthermore, lumbar muscle activation during isometric testing and at the start of the

24 dynamic fatigue test was unexpectedly increased and patients surprisingly demonstrated 
1 greater muscle fatigability (assessed via the rate of median frequency decline) post-

2 therapy. The authors suggested that patients might be employing different motor

3 control/recruitment patterns after treatment, perhaps as a result of less utilisation of

4 guarding mechanisms. This may help to explain the findings in the current study.

5 Following the programme, patients may have been using painful lumbar muscles to

6 maintain upright posture to a greater extent than previously, or adopting an altered

7 posture, such as a more neutral spine, leading to changes in muscle activation patterns.

8 This suggests that elevated muscle activity may not necessarily be problematic in the

9 short-term and may sometimes reflect a positive adjustment. This should be borne in

10 mind when considering the use of techniques such as EMG biofeedback that aim to

11 encourage decreased EMG levels.

12

13 Although both absolute and normalised EMG levels were reduced by the follow-up

14 session, in neither case was this significant (possibly due to the small sample size). Over

15 this six-month period, changes in muscle activity were associated with changes in each of

16 disability, catastrophising, pain-related anxiety, depression and self-efficacy. Although

17 based on limited numbers, these results are consistent with the findings of a cross-sectional

18 study which found significant correlations between muscle activity and each of these

19 variables (Lewis et al., 2012). This earlier study also found muscle activity to be a

20 mediating factor between psychological factors and pain. Together, the results of both

21 studies confirm the link between biomechanical and psychological factors in CLBP and add

22 support to the importance of muscle activity within CLBP, although more research is

23 required to fully understand the mechanisms and relationships involved. 
1 Contrary to our hypothesis, the results did not support a correlation between changes in

2 stature recovery and muscle activity following the rehabilitation programme. This study

3 had the advantage of deriving data from a clinical sample with moderate levels of pain

4 and disability and the results suggest that the relationship between muscle activity and

5 stature recovery within this patient population may be more complex than originally

6 thought. For example, there may have been changes in the patterns of paraspinal EMG

7 that were more complicated or occurring at a deeper level than could be detected with

8 only four sites of superficial EMG. Alternatively, there may simply have been too many

9 confounding factors when comparing these measurements over several weeks or months

10 in a fluctuating condition such as CLBP, particularly with the added complication of

11 varied treatment responses.

12

13 The assessment of muscle activity was carried out while the patients were in a static

14 standing posture as it is commonly reported that patients with CLBP have elevated

15 paraspinal muscle activity in this position (Geisser et al., 2005). Although studies, such

16 as Mannion et al. (2001), have assessed muscle activity during movement or strength

17 and endurance tasks, it was decided not to include a dynamic assessment in the current

18 study due to the severity of the condition of some of the patients. The average disability

19 of participants in the current study was higher than the group assessed by Mannion et al.

20 (initial RDQ of 12.0 (SD 4.9) compared to 7.8 (SD 4.6) respectively) and included some

21 patients with severe back pain who would have been unable or unwilling to perform

22 dynamic tasks, particularly at the initial visit.

24 Limitations 
1 There were some limitations to our study. Many of the patients were taking analgesics

2 and some patients changed their medication use during the course of the study, possibly

3 as a result of advice given within the rehabilitation programs, which may have affected

4 the pain scores in particular. It was also not possible to control the spinal loading that

5 occurred prior to the participants attending each testing session and this may therefore

6 have varied between both participants and visits; however the impact of this may have

7 been mitigated by the EMG preparation and baseline measurements which formed a

8 standardised activity at the start of each session. Finally, the sample size for the follow-

9 up session in particular was smaller than we would have wished, which limited the

10 statistical power of the analysis. Nevertheless, the results still showed a number of

11 interesting findings, including a highly significant increase in stature recovery over this

12 six-month period. We recommend that these findings are confirmed with a larger

13 sample size. It would additionally be interesting to see if patients with acute or sub-

14 acute LBP demonstrate the same pattern of results as the CLBP population considered

15 in the current study.

17 Conclusions

18 In conclusion, the increased rate of stature recovery by the six-month follow-up

19 suggests that the delayed recovery seen in patients with CLBP is not primarily the result

20 of disc degeneration. Furthermore, an immediate decrease in EMG levels following

21 active treatment may not always be the optimal response for long-term improvements in

22 clinical outcome and a period of adaptation might be expected. 


\section{$1 \quad \underline{\text { References }}$}

2 Adams MA, Bogduk N, Burton K, Dolan P. The Biomechanics of Back Pain.

3 Edinburgh: Churchill Livingstone, 2002.

4 Adams MA, Dolan P, Hutton WC, Porter RW. Diurnal changes in spinal mechanics and 5 their clinical significance. J Bone Joint Surg Br 1990;72-B:266-70.

6 Ambroz C, Scott A, Ambroz A, Talbott EO. Chronic low back pain assessment using

7 surface electromyography. J Occup Environ Med 2000;42:660-9.

8 Anderson KO, Dowds BN, Pelletz RE, Edwards WT. Peeters-Asdourian C.

9 Development and initial validation of a scale to measure self-efficacy beliefs in patients

10 with chronic pain. Pain 1995;63:77-84.

11 Coons MJ, Hadjistavropoulos HD, Asmundson GJ. Factor structure and psychometric

12 properties of the Pain Anxiety Symptoms Scale-20 in a community physiotherapy clinic 13 setting. Eur J Pain 2004;8:511-6.

14 Dankaerts W, O’Sullivan PB, Burnett AF, Straker LM, Danneels LA. Reliability of

15 EMG measurements for trunk muscles during maximal and sub-maximal voluntary 16 isometric contractions in healthy controls and CLBP patients. J Electromyogr Kinesiol $17 \quad 2004 ; 14: 333-42$.

18 Geisser ME, Ranavaya M, Haig AJ, Roth RS, Zucker R, Ambroz C, et al. A meta-analytic 19 review of surface electromyography among persons with low back pain and normal, healthy 20 controls. J Pain 2005;6:711-26. 
1 Healey EL, Fowler NE, Burden A, McEwan IM, Raised paraspinal muscle activity

2 reduces rate of stature recovery after loaded exercise in individuals with chronic low

3 back pain. Arch Phys Med Rehab 2005;86:710-5.

4 Hupli M, Heinonen R, Vanharanta H. Height changes among chronic low back pain

5 patients during intense physical exercise. Scand J Med Sci Sports 1997;7:32-7.

6 Jensen MP. The validity and reliability of pain measures for use in clinical trials in

7 adults: review paper written for the Initiative on Methods, Measurement, and Pain

8 Assessment in Clinical Trials (IMMPACT-II) meeting. 2003 Apr 12-13; Washington,

9 US.

10 Kori SH, Miller RP, Todd DD. Kinisophobia: a new view of chronic pain behaviour.

11 Pain Manag 1990;3:35-43.

12 Lewis SE. The relationships between stature recovery, muscle activity and

13 psychological factors in patients with chronic low back pain [doctoral thesis]. Crewe:

14 Manchester Metropolitan Univ; 2011.

15 Lewis SE, Fowler NE. Changes in intervertebral disk dimensions after a loading task

16 and the relationship with stature change measurements. Arch Phys Med Rehabil

$17 \quad 2009 ; 90: 1795-9$.

18 Lewis S, Holmes P, Woby S, Hindle J, Fowler N. The relationships between measures

19 of stature recovery, muscle activity and psychological factors in patients with chronic

20 low back pain. Man Ther 2012;17:27-33. 
1 Mannion AF, Taimela S, Müntener M, Dvorak J. Active therapy for chronic low back

2 pain: Part 1. Effects on back muscle activation, fatigability, and strength. Spine

$3 \quad 2001 ; 26: 897-908$.

4 Panjabi M. The stabilizing system of the spine. Part II. Neutral zone and instability

5 hypothesis. J Spinal Disord 1992;5:390-7.

6 Rodacki CL, Fowler NE, Rodacki AL, Birch K. Stature loss and recovery in pregnant

7 women with and without low back pain. Arch Phys Med Rehabil 2003;84:507-12.

8 Roland M, Fairbank J. The Roland-Morris Disability Questionnaire and the Oswestry

9 Disability Questionnaire. Spine 2000;25:3115-24.

10 Roland M, Morris R. A study of the natural history of low back pain: part 1.

11 Development of a reliable and sensitive measure of disability in low-back pain. Spine $12 \quad 1983 ; 8: 141-4$

13 Shan X, Zhang Y, Zhang T, Chen Z, Wei Y. Flexion relaxation of erector spinae

14 response to spinal shrinkage. J Electromyogr Kinesiol 2012;22:370-5.

15 Sullivan MJL, Bishop SR, Pivik J. The Pain Catastrophizing Scale: development and 16 validation. Psychol Assess 1995;7:524-32.

17 Turner JA, Fulton-Kehoe D, Franklin G, Wickizer TM, Wu R. Comparison of the

18 Roland-Morris Disability Questionnaire and generic health status measures: a

19 population-based study of workers' compensation back injury claimants. Spine

$20 \quad 2003 ; 28: 1061-7$.

21 Urban JPG, Roberts S. Degeneration of the intervertebral disc. Arthritis Res Ther $22 \quad 2003 ; 5: 120-30$. 
1 van Dieën JH, Selen LPJ, Cholewicki J. Trunk muscle activation in low-back pain

2 patients, an analysis of the literature. J Electromyogr Kinesiol 2003;13:333-51.

3 Watson PJ, Booker CK, Main CJ. Evidence for the role of psychological factors in

4 abnormal paraspinal activity in patients with chronic low back pain. J Musculoskelet

5 Pain 1997a;5:41-56.

6 Watson PJ, Booker CK, Main CJ, Chen ACN. Surface electromyography in the

7 identification of chronic low back pain patients: the development of the flexion

8 relaxation ratio. Clin Biomech 1997b;12:165-71.

9 Woby SR, Roach NK, Urmston M, Watson PJ. Outcome following a physiotherapist-

10 led intervention for chronic low back pain: the important role of cognitive processes.

11 Physiother 2008;94:115-24.

12 Zhao F, Pollintine P, Hole BD, Dolan P, Adams MA. Discogenic origins of spinal

13 instability. Spine 2005;30:2621-30.

14 Zigmond AS, Snaith RP, 1983. The Hospital Anxiety and Depression Scale. Acta

15 Psychiatr Scand 1983;67:361-70.

16 


\section{Captions to illustrations}

2 Figure 1. Participant in position in the stadiometer 